\title{
Effectiveness of Oral Tofacitinib Dose Tapering in a Case of Alopecia Areata Universalis
}

\author{
Mariana Esteves ${ }^{a} \quad$ Sofia Lopes $^{a} \quad$ Filomena Azevedo $^{a} \quad$ Ana Pedrosa ${ }^{a}$ b \\ aServiço de Dermatologia e Venereologia, Centro Hospitalar Universitário de São João EPE, Porto, Portugal; \\ ${ }^{\mathrm{b}}$ Faculdade de Medicina, Universidade do Porto, Porto, Portugal
}

\section{Established Facts}

- Alopecia areata (AA) is a form of nonscarring hair loss, characterized by alopecic patches that, in its most severe variants, can encompass the entire scalp or body.

- The currently employed therapies are neither approved nor reliably effective for treatment of AA.

\section{Novel Insights}

- Janus kinase inhibitors have demonstrated the ability to reverse the disease.

- Tofacitinib is an effective and safe modality for the management of alopecia areata and should be considered in severe, refractory disease.

\section{Keywords}

Hair disorder - Alopecia areata · Janus kinase inhibitor .

Tofacitinib

\section{Abstract}

Introduction: Alopecia areata $(\mathrm{AA})$ is a form of nonscarring alopecia and one of the most common autoimmune disorders. Persistent or severe variants lead to potential disfigurement and are associated with a significant negative impact on the patient's quality of life. Case Presentation: A 51-yearold female patient presenting with refractory alopecia universalis was successfully treated with oral tofacitinib. Recurrence was not observed following 17 months of therapy. Discussion/Conclusion: None of the currently employed therapies for AA are reliably effective nor are they approved for the disease. In this setting, Janus kinase inhibitors emerge as a promising novel treatment, as increasing evidence supports their effectiveness in AA.

(c) 2020 S. Karger AG, Basel

\section{Introduction}

Alopecia areata (AA) is one of the most common autoimmune disorders, with an estimated lifetime risk of $1.7 \%[1,2]$. It is a nonscarring alopecia, resulting from the loss of hair follicle immune privilege, which leads to hair cycle growth arrest in the anagen phase [3]. Variants of AA include alopecia totalis (AT), in which all scalp hair is lost, and alopecia universalis (AU), characterized by complete loss of both scalp and body hair. Currently em- 


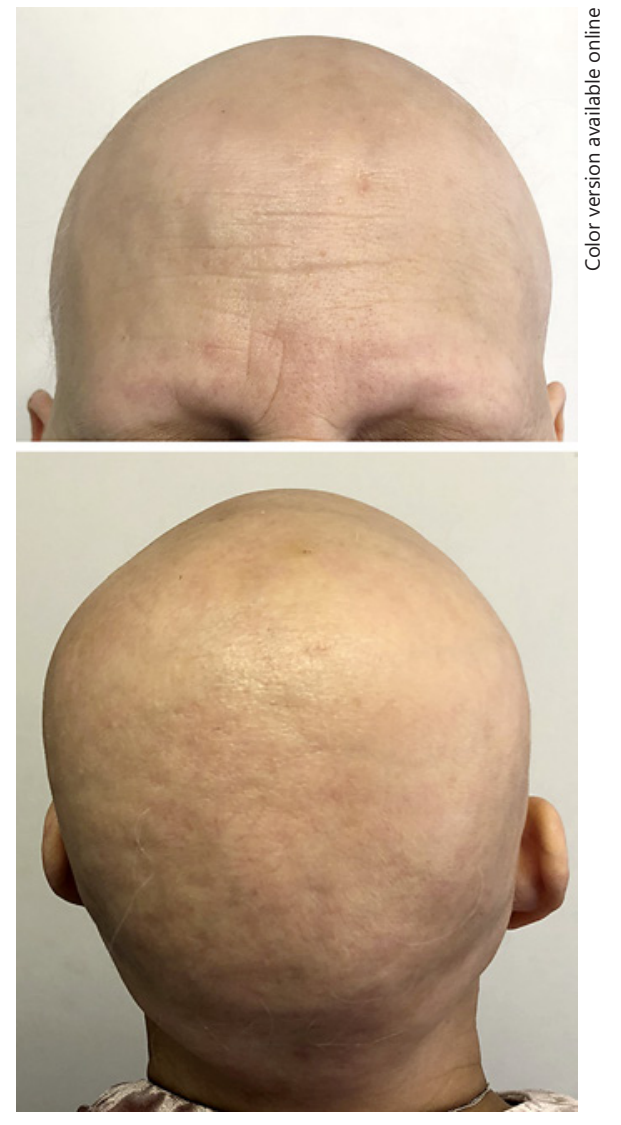

Fig. 1. Baseline photographs of our patient, showing a complete absence of eyebrows and scalp hair (SALT score 100). SALT, Severity of Alopecia Tool.

ployed therapies for AA include corticosteroids, minoxidil, diphenylcyclopropenone, and immunosuppressants, such as cyclosporine or methotrexate [4]. Nevertheless, none of these are reliably effective, particularly in severe cases, nor are they approved for the disease [1-3]. In this setting, tofacitinib and other Janus kinase (JAK) inhibitors are yielded as a promising novel treatment, as increasing evidence suggests that they may be of therapeutic use for AA.

\section{Case Report}

A 51-year-old female patient presented to our department with a 3-year history of AA that had progressed to AU 1 year prior. Her previous medical history was relevant for arterial hypertension, as well as medicated and controlled hypothyroidism, following total thyroidectomy due to multinodular goiter. Previous treatments included topical and intralesional corticosteroids, topical minoxidil $5 \%$, and multiple courses of oral prednisolone (up to $1 \mathrm{mg} / \mathrm{kg}$ per

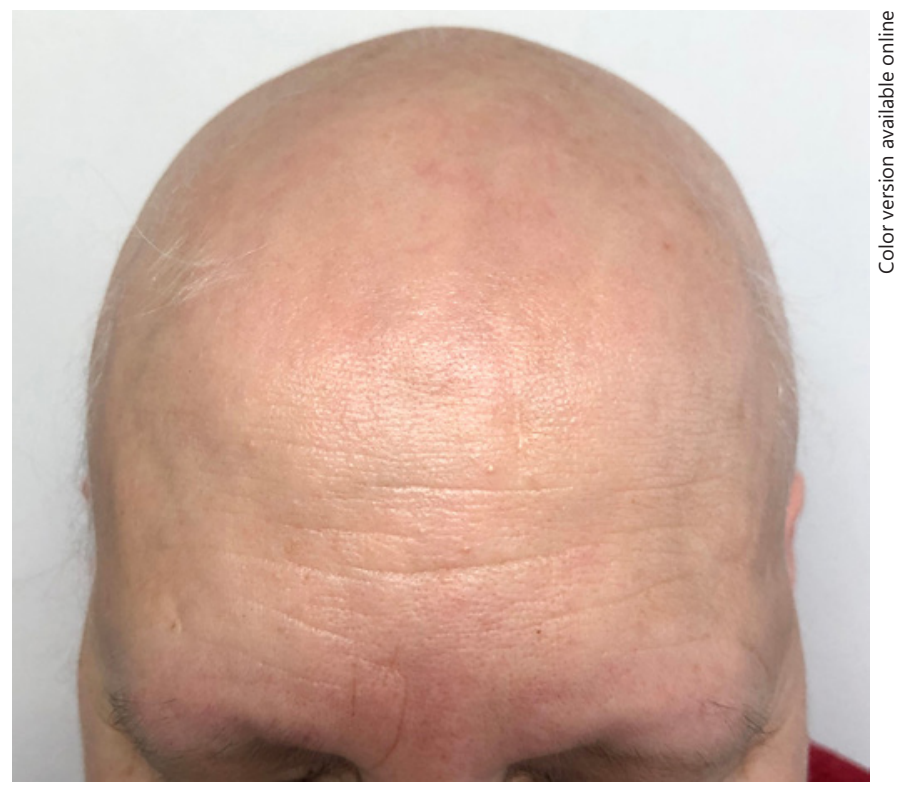

Fig. 2. At 6 weeks of treatment. Presence of fine vellus hair on the scalp and partial eyebrow regrowth can be noted.

day), without significant improvement. Topical immunotherapy with diphenylcyclopropenone $0.5 \%$ was also attempted for 15 months, without clinical response.

Physical examination revealed complete absence of hair on the scalp and eyebrows, along with sparse body hair (Fig. 1). Given the clinical picture, the patient was proposed to treatment with oral tofacitinib. Baseline investigation included a complete blood count; liver, renal, and thyroid functions; serologic markers for human immunodeficiency virus and viral hepatitis B and C; chest radiograph; tuberculin skin test; and interferon gamma release assay. No significant abnormalities were found, except for elevated hepatitis B anti-core antibody titers, along with anti-surface antibody titers $>1,000 \mathrm{UI} / \mathrm{L}$. In light of this, the patient was referred to an infectologist. Additional studies revealed a negative hepatitis B surface antigen and undetected viral DNA by PCR.

As such, oral tofacitinib was initiated at $5 \mathrm{mg}$ twice daily. Response to treatment and hair regrowth was evaluated using Severity of Alopecia Tool (SALT) score. The initial SALT score for our patient was 100, as a complete absence of hair was noted. After 6 weeks of treatment, fine vellus hair was noted, along with partial eyebrow regrowth (Fig. 2). Following 5 months of therapy, significant hair regrowth was seen on the scalp and eyebrows, and the SALT score was 13 (Fig. 3). By 8 months of treatment, there was complete hair regrowth, with a SALT score of 4 (Fig. 4). Tofacitinib was then reduced to $5 \mathrm{mg}$ daily at once for 5 months and to $5 \mathrm{mg}$ twice weekly at the latest follow-up, without evidence of recurrence. At this point, oral minoxidil $1 \mathrm{mg}$ daily was also added to the regimen. No new alopecic patches have developed throughout the course of treatment, and no serious adverse side effects were observed at any stage. No laboratory abnormalities were evident, and hepatitis B viral DNA remained undetected by serial PCR analysis. 
3
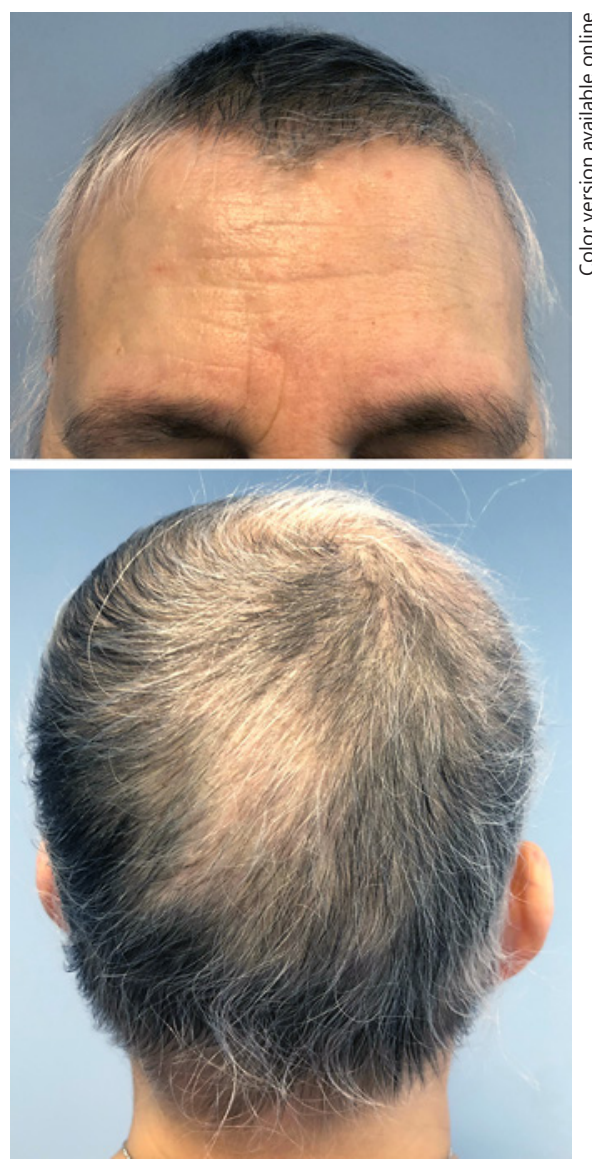

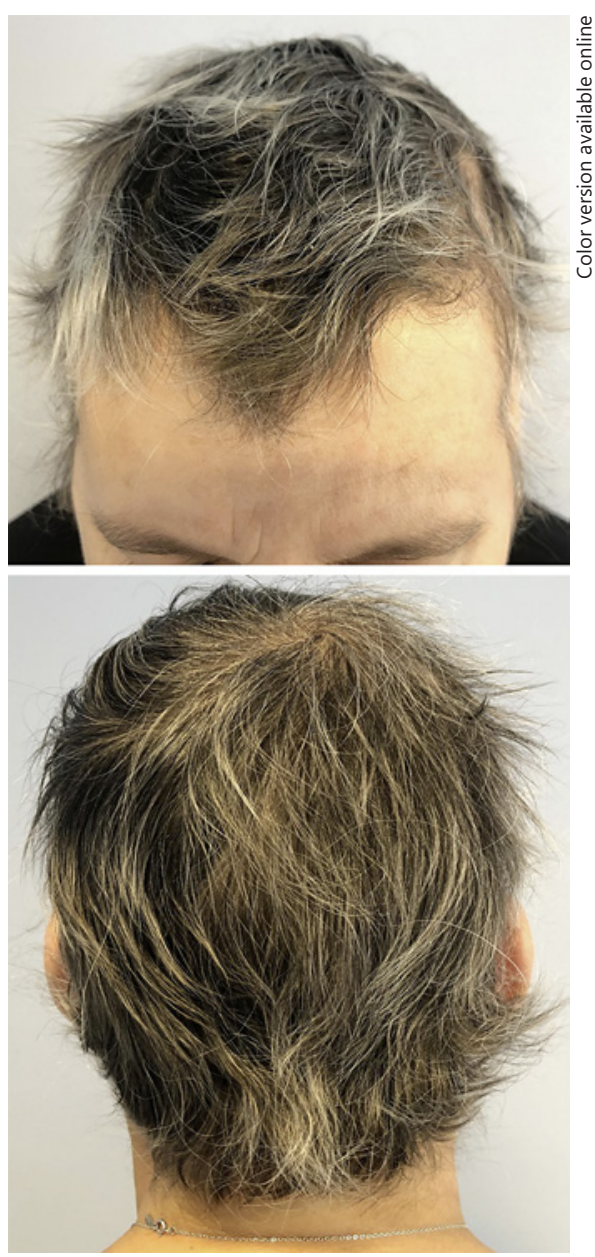

Fig. 3. At 5 months of treatment. Significant hair regrowth can be seen on the scalp and eyebrows (SALT score 13). SALT, Severity of Alopecia Tool.

Fig. 4. At 8 months of treatment with tofacitinib $10 \mathrm{mg} /$ daily. Complete hair regrowth can be observed (SALT score 4). SALT, Severity of Alopecia Tool.

\section{Discussion}

Recent studies regarding the pathophysiology of AA have concluded that autoreactive $\mathrm{CD}^{+} \mathrm{T}$ lymphocytes play a key role in its development. These cells were shown to release interferon-gamma, which causes upregulation of interleukin-15 via JAK/STAT signaling. Amplification of the inflammatory response around hair follicles ultimately leads to their damage and to clinical hair loss [1, $2,4]$. Therefore, blockade of JAK-dependent activation of cytokine signaling pathways was demonstrated to be effective in reversing the disease [1-3].

Tofacitinib is a selective JAK 1 and 3 inhibitor, currently approved for treatment of refractory rheumatoid arthritis [5-7]. Improvement of AA was first noted in a patient with $\mathrm{AU}$ and psoriasis, who was being treated with tofacitinib [8]. Since then, an increasing number of studies and case reports support the potential effectiveness of JAK inhibition for AA, even in severe forms such as AT or AU [1-14]. More than $70 \%$ of patients show some degree of response [4]. An overall good or complete response rate, defined as a greater than $50 \%$ improvement in SALT score, is observed in up to $54 \%$ of patients treated with tofacitinib [10]. Our case supports the previously published literature, as complete hair regrowth was achieved after 8 months of therapy.

In most studies, oral tofacitinib is initiated as monotherapy at a standard dose of $5 \mathrm{mg}$ twice daily [1-11]. A recently published article evaluated the efficacy of lowdose (5 $\mathrm{mg}$ daily) tofacitinib and found that this regimen might be efficient and cost-saving in a proportion of cases [12]. Our patient has been treated with tofacitinib for 17 months and remains without recurrence following dose tapering. However, in nonresponders or in those who experience a flare during treatment, dose escalation to $10 \mathrm{mg}$ twice daily or association with oral corticosteroids might be necessary in order to achieve clinical improvement $[1,2]$. Combination therapy of tofacitinib and 
oral minoxidil has also been suggested and was found to be more efficacious in some cases than tofacitinib monotherapy, particularly in nonresponders [13]. In comparison with topical preparations, oral JAK inhibitors appear to be associated with a higher chance of achieving a good clinical response, regardless of the pharmacological agent used $[4,14]$.

Initial hair growth in responders can be seen as soon as 4 weeks after treatment initiation [11], while complete hair regrowth is observed following an average of 6.7 months [4]. Recommended duration of treatment is not yet established, although a mean duration of at least 12 months is reported in most trials [1,4-7]. Despite the promising outcomes, lack of durability of effect is commonly described $[1,3-6,9,10]$. Relapse has been shown to occur in up to 8.5 weeks [3] or sometimes even earlier [5], mainly after discontinuation of treatment. Also, a rebound effect has been described, in which worsening of the baseline alopecia occurs $[11,15]$. Subsequent to relapse, hair regrowth can again be achieved [1], which suggests that maintenance therapy is necessary for continuous remission $[1,9,10]$.

Earlier reports state that the duration of the current episode of AA is one of the most important predictors of effectiveness $[1,3,9]$, as after 10 years of hair loss, improvement is much less likely to occur [1]. Likewise, worst treatment outcomes were noted in patients with AT or AU $[1,3]$. Nevertheless, recent evidence suggests that neither disease severity nor duration seems to greatly influence response to tofacitinib $[4,5,11,14]$. Similarly, demographic factors, such as age or gender, do not appear to be useful predictors [4], while oral dose and duration of treatment may be the most important factors influencing the therapeutic effect [10].

Oral tofacitinib is generally well-tolerated. Reported adverse effects are mostly mild and include upper respiratory tract infections, headache, and acne $[1,3,10]$. Laboratory abnormalities, if present, include mild transaminitis, cytopenias, and lipid profile alterations $[4,10]$. Although data regarding the safety profile of tofacitinib in patients with AA are limited, no cases of serious infections, malignancies, or reactivation of tuberculosis were reported $[1,4,9,14]$. Still, there appears to be an increased risk of viral reactivation, particularly herpes zoster $[4,5]$. Our patient was closely monitored in collaboration with an infectologist regarding the history of previous hepatitis B; however, there was no evidence of reactivation during the course of treatment. Evaluation of challenging cases, such as our own, by multidisciplinary teams is, therefore, mandatory.

Oral Tofacitinib in Alopecia Areata

\section{Conclusion}

Persistent AA or its severe variants lead to important disfigurement and are associated with a significant negative impact on the quality of life, as well as high rates of anxiety and depression $[1,2,4]$. Hence, new therapeutic developments are of crucial importance. While tofacitinib is not a cure for AA, as relapse after discontinuation frequently occurs, it has been demonstrated to be a potentially effective and safe modality for the management of severe, refractory disease. Nevertheless, available data were derived mostly from low-quality, non-comparative studies, mainly case reports or small case series $[4,10]$. As such, further larger sized, randomized controlled trials are needed, so as to define the optimal treatment protocol for maintenance of response and minimization of risks.

\section{Statement of Ethics}

The patient has given consent to publish details and photographs of the case.

\section{Conflict of Interest Statement}

The authors have no conflicts of interest to declare.

\section{Funding Sources}

The authors have no funding sources to declare.

\section{Author Contributions}

All named authors met the International Committee of Medical Journal Editors (ICMJE) criteria for authorship of this article and gave approval for this version to be published.

References

1 Liu LY, Craiglow BG, Dai F, King BA. Tofacitinib for the treatment of severe alopecia areata and variants: a study of 90 patients. J Am Acad Dermatol. 2017 Jan;76(1):22-8.

2 Chiang A, Ortenzio F, Juhasz MLW, Yu V, Mesinkovska NA. Balance of tofacitinib efficacy and disease flare in the treatment of alopecia universalis: a case report and review of the literature. JAAD Case Rep. 2018 Aug;4(7):733-6.

3 Kennedy Crispin M, Ko JM, Craiglow BG, Li S, Shankar G, Urban JR, et al. Safety and efficacy of the JAK inhibitor tofacitinib citrate in patients with alopecia areata. JCI Insight. 2016 Sep;1(15):e89776. 
4 Phan K, Sebaratnam DF. JAK inhibitors for alopecia areata: a systematic review and metaanalysis. J Eur Acad Dermatol Venereol. 2019 May;33(5):850-6.

5 Hogan S, Wang S, Ibrahim O, Piliang $M$, Bergfeld W. Long-term treatment with tofacitinib in severe alopecia areata: an update. J Clin Aesthet Dermatol. 2019 Jun;12(6):12-4.

6 Ibrahim O, Bayart CB, Hogan S, Piliang M, Bergfeld WF. Treatment of alopecia areata with tofacitinib. JAMA Dermatol. 2017 Jun; 153(6):600-2.

7 Serdaroglu S, Engin B, Çelik U, Erkan E, Askin O, Oba Ç, et al. Clinical experiences on alopecia areata treatment with tofacitinib: a study of 63 patients. Dermatol Ther. 2019 May;32(3):e12844.
8 Craiglow BG, King BA. Killing two birds with one stone: oral tofacitinib reverses alopecia universalis in a patient with plaque psoriasis. J Invest Dermatol. 2014 Dec;134(12):2988-90.

9 Park HS, Kim MW, Lee JS, Yoon HS, Huh $\mathrm{CH}$, Kwon $\mathrm{O}$, et al. Oral tofacitinib monotherapy in Korean patients with refractory moderate-to-severe alopecia areata: a case series. J Am Acad Dermatol. 2017 Nov;77(5): 978-80.

10 Guo L, Feng S, Sun B, Jiang X, Liu Y. Benefit and risk profile of tofacitinib for the treatment of alopecia areata: a systemic review and meta-analysis. J Eur Acad Dermatol Venereol. 2020 Jan;34(1):192-201.

11 Jabbari A, Sansaricq F, Cerise J, Chen JC, Bitterman A, Ulerio G, et al. An open-label pilot study to evaluate the efficacy of tofacitinib in moderate to severe patch-type alopecia areata, totalis, and universalis. J Invest Dermatol. 2018 Jul;138(7):1539-45.
12 Chen YY, Lin SY, Chen YC, Yang CC, Lan CE. Low-dose tofacitinib for treating patients with severe alopecia areata: an efficient and cost-saving regimen. Eur J Dermatol. 2019 Dec;29(6):667-9.

13 Wambier CG, Craiglow BG, King BA. Combination tofacitinib and oral minoxidil treatment for severe alopecia areata. J Am Acad Dermatol. 2019 Sep 6;S0190-9622(19)32688$\mathrm{X}$.

14 Park H, Yu DA, Kwon O. Janus kinase inhibitors: an innovative treatment for alopecia areata. J Dermatol. 2019 Aug;46(8):724-30.

15 Gordon SC, Abudu M, Zancanaro P, Ko JM, Rosmarin D. Rebound effect associated with JAK inhibitor use in the treatment of alopecia areata. J Eur Acad Dermatol Venereol. 2019 Apr;33(4):e156-7. 